\title{
Aspectos de la seguridad del paciente. Gestión y prevención de riesgos en el quirófano de dermatología
}

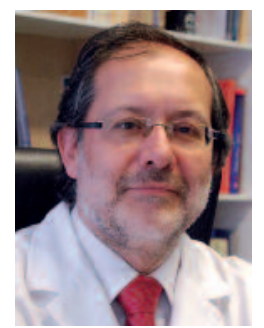

Francisco Leyva Rodríguez Jefe de Servicio de Cirugía Plástica, Estética y

Reparadora. Hospital Universitario Clínico San Carlos. Madrid.

Profesor asociado. Universidad Complutense. de Madrid.
Los efectos o acontecimientos adversos documentados en los hospitales públicos pueden llegar a presentar, dependiendo de las series publicadas, una incidencia de entre el $35 \%$ y el $43 \%$, y se considera que en el $17,7 \%$, aproximadamente, de los pacientes condicionarán discapacidad o muerte. En el Estudio Nacional de Efectos Adversos (ENEAS $)^{1}$, impulsado y financiado por el Ministerio de Sanidad, se demuestra que el $8,4 \%$ de los pacientes ingresados en los hospitales del Sistema Nacional de Salud sufren alguna forma de efecto adverso relacionado con la asistencia recibida durante su estancia en el centro. Entre estas reacciones, la mayor parte, un $37,4 \%$, estaban relacionadas con la utilización de fármacos, un $25,3 \%$ con las infecciones hospitalarias y un $25 \%$ con la aplicación de procedimientos quirúrgicos, sobre todo cuando requieren anestesia. Asimismo, un 4,4\% de dichos hospitalizados fallece. Por otra parte, también sabemos que el riesgo aumenta con la duración de la estancia $(6 \%$ por día de ingreso) y con otros factores como la edad del paciente.

Consideramos como gestión y prevención de riesgos el conjunto de actividades destinado tanto a evitar (o reducir) el riesgo de que se produzca un efecto adverso durante la asis- tencia como a disminuir sus consecuencias negativas; ello debe ser un objetivo prioritario en los servicios quirúrgicos.

Varios estudios demuestran la influencia de factores organizativos y de dirección en el sistema de reducción del riesgo asistencial y en el resultado de los enfermos quirúrgicos, como lo demuestra el informe denominado «Bloque Quirúrgico», editado por el Ministerio de Sanidad ${ }^{2}$. Los sistemas de registro y notificación voluntarios y anónimos permiten identificar un importante porcentaje de estos incidentes, analizar los factores relacionados (que contribuyen o limitan) y establecer estrategias preventivas, lo que permite gestionar el riesgo y reducir potencialmente la aparición y las consecuencias de los efectos adversos evitables. Iniciativas como el ICU Safety Reporting System

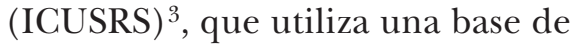
datos web como sistema de registro e incluye aportaciones de diferentes centros, favorecen la cultura de la seguridad y del riesgo, imprescindibles en la mejora de la calidad asistencial de los enfermos graves.

Quiero desde estas líneas proponer una serie de mejoras que sin duda beneficiarán la práctica clínica habitual de un servicio médico-quirúrgico como lo es la dermatología. Para 
ello seguiré los siguientes pasos, conociendo que los riesgos se producen siguiendo un modelo matemático de sistemas independientes y que no son tanto debidos a errores activos o vinculados a las personas como a condiciones latentes (ambientales...) resolubles por la alta dirección: en primer lugar, la identificación de sistemas no seguros; a continuación, la introducción de barreras o pantallas en función de la evaluación previa, la frecuencia de presentación, la gravedad de esta y las condiciones latentes. La tercera fase es la de tratamiento mediante la prevención, administración y financiación.

Una forma de prevenir es identificar el incidente, entendiendo como tal las situaciones en las que ha podido pasar algo y no ha pasado; se estima que para que se produzca un efecto adverso este habrá avisado (incidente) 300 veces. Después se debe poner en marcha un análisis de mejora, pues es fundamental buscar la causa y no quedarse en la mera consecuencia, ya que de la primera podremos buscar soluciones.

Entre las formas de identificación emplearemos las clásicas preguntas: ¿Qué ha pasado? ¿Por qué ha pasado? y ¿qué hacer para evitarlo? A continuación pondremos en marcha un programa de formas de identificación que puede estar basado en listados, listas específicas, registros-documentación, entrevistas, bibliografía y otras herramientas de calidad.

En esta línea cabe destacar tres acciones que nos pueden ayudar: en primer lugar, las puestas en marcha por el Ministerio de Sanidad y Política Social basadas en estudios de incidencia, la formación de profesionales en técnicas de gestión de riesgos, los sistemas de identificación inequívoca de pacientes ingresados y la bioseguridad y disminución de infecciones nosocomiales. En segundo lugar, las herramientas de mejora continua tipo ARC-AMFE, donde ARC es el análisis de causa raíz y AMFE el análisis modal de causas (fallos)-efectos. Y, en tercer lugar, las recomendaciones ENEAS y el proyecto IDEA (identificación de efectos adversos).

Con todo ello, propongo un programa de trabajo que debe incluir: «Programa de cirugía segura» mediante la implantación del denominado check-list en el quirófano. Programa de higiene de manos, con la implantación y desarrollo del mismo. Programa de farmacovigilancia, con la extensión del uso de dispositivos de dispensación de fármacos (SADME), como el Pyxis, OMNICELL o KRZ. Y, finalmente, la elaboración de mapas de riesgos y el registro de incidentes críticos.

La aplicación de todas o una parte de estas medidas de forma progresiva y escalonada redundará, sin duda, en una mayor seguridad para el paciente en el entorno de un hospital del siglo XXI y en concreto en el quirófano de dermatología.

\section{BIBLIOGRAFÍA}

1. Ministerio de Sanidad y Consumo. Estudio Nacional sobre los Efectos Adversos ligados a la Hospitalización. ENEAS 2005. Madrid: Ministerio de Sanidad y Consumo; 2006. Disponible en: http://www.msssi.gob.es/organizacion/sns/planCalidad SNS/pdf/excelencia/opsc_sp2.pdf

2. Ministerio de Sanidad y Política Social. Bloque Quirúrgico. Estándares y recomendaciones. Madrid: Ministerio de Sanidad y Política Social; 2010. Disponible en: http://www.msssi.gob.es/ organizacion/sns/planCalidadSNS/docs/BQ.pdf

3. Holzmueller CG, Pronovost PJ, Dickman F, Thompson DA, Wu AW, Lubomski LH, et al. Creating the web-based intensive care unit safety reporting system. J Am Med Inform Assoc. 2005;12(2):130-9. 